\title{
DESARROLLO E IMPLEMENTACIÓN DE UNA NORIA MODIFICADA COMO PROPUESTA SOSTENIBLE DE RECIRCULACIÓN DE AGUA PARA UNA ESTACIÓN PISCÍCOLA
}

\author{
Christine M. Hahn-von-Hessberg ${ }^{1}$ \\ Herbert E. Quintero ${ }^{2}$ \\ Alberto Grajales-Quintero ${ }^{3}$
}

Recibido el 24 de junio de 2014, aprobado el 1 de abril de 2015 y actualizado el 06 de noviembre de 2015

DOI: 10.17151/luaz.2016.42.12

\section{RESUMEN}

Existen problemas de abastecimiento de agua en verano e invierno, en la Estación Piscícola de la Universidad de Caldas (Colombia). El trabajo muestra la manera como, por medio de un sistema de recirculación de agua, se provee a la Estación del líquido, de manera persistente, conservando parámetros fisicoquímicos aptos para el cultivo de peces.

Cambios en el uso del suelo, como desarrollos urbanísticos, han generado impacto sobre la microcuenca que abastece la Estación. Surge la propuesta de reutilizar el agua mediante un modelo sostenible, adaptando un mecanismo ancestral: la Noria. Éste trabajo se desarrolló en tres etapas: recopilación de información, seguimiento e implementación de la propuesta. Para el montaje se requirió adaptar parte de la infraestructura existente de la Estación para recolectar el agua. El diseño de la Noria consta de un sistema de captación de agua (Cilindro con compartimentos) que impulsa un sistema de poleas y a su vez a la Noria modificada con canjilones, la cual capta agua $(1,5 \mathrm{l} / \mathrm{seg})$ y la elevan $7 \mathrm{~m}$. Los parámetros físicoquímicos (temperatura, oxígeno y $\mathrm{pH}$ ) y biológicos (macroinvertebrados acuáticos) permanecieron en rangos normales para un cultivo de peces tropicales. El diseño realizado es modular, no requiere energía eléctrica, ni mano de obra especializada para su mantenimiento, puede ser utilizado como una herramienta alterna en el sector agropecuario particularmente en el sector acuícola, donde se presenten dificultades hídricas, es de bajo costo y ambientalmente sostenible.

\section{PALABRAS CLAVE}

Rueda hidráulica, piscicultura, microcuenca hidrográfica, abastecimiento hídrico, sostenibilidad.

\section{DEVELOPMENT AND IMPLEMENTATION OF A MODIFIED WATERWHEEL AS A PROPOSAL FOR SUSTANTAINABLE WATER CIRCULATION FOR A FISHING STATION.}

\section{ABSTRACT}

There are problems of water supply in the summer and the winter, in the Fishing Station of Universidad de Caldas (Colombia). This article shows how, by means of a water recirculation system, the Station is provided with the liquid persistently, keeping physicochemical parameters fit for fish farming.

Changes in land use, such as urban development, have generated impact on the watershed that supplies the Station. Then, a proposal to reuse 
water through a sustainable model, adapting an ancestral mechanism surges: the Waterwheel. This work was developed in three stages: data collection, monitoring and implementation of the proposal. For the assembly adaption of part of the existing infrastructure of the Station to collect water was required. The design of the waterwheel consists of a water catchment system (cylinder with compartments) that drives a pulley system and in turn to the modified waterwheel with buckets, which capture the water $(1.5 \mathrm{I} / \mathrm{sec})$ and then raise it $7 \mathrm{~m}$. The physicochemical parameters (temperature, oxygen and $\mathrm{pH}$ ) and biological (macroinvertebrate) remained within normal ranges for a crop of tropical fish. The design developed is modular, it does not require electricity or skilled workforce for maintenance, it can be used as an alternative tool in agriculture particularly in the aquaculture sector where water difficulties arise, and it is inexpensive and environmentally sustainable .

KEY WORDS: Water wheel, fish farming, hydrographic watershed, water supply, sustainability.

\section{INTRODUCCIÓN}

Colombia es catalogada como uno de los países con mayor oferta hídrica del mundo, sin embargo al realizar un análisis crítico de la oferta y demanda del agua se observa el desbalance del mismo; posee 6 vertientes, destacándose la vertiente Caribe, compuesta por las cuencas del río Magdalena, Cauca y Sinú, el cual agrupa el $70 \%$ de la actividad económica, social e industrial del país. Con una precipitación anual de $2000 \mathrm{~mm} / a n ̃ o$, una escorrentía del $30 \%$ con valores medios de $1000 \mathrm{~mm}$ en la región Magdalena-Cauca y $1500 \mathrm{~mm}$ para el Medio Cauca (MAVDT y Viceministerio de Ambiente, 2010). Panorama hídrico cambiante como consecuencia de la variabilidad climática, con incrementos y disminuciones en algunas regiones hasta de un 15\% (MAVDT, 2010; Quintero-Ángel, Carvajal-Escobar y Aldunce, 2012), incluyendo al departamento de Caldas; CORPOCALDAS (2006) reporta que más del $70 \%$ de las fuentes hídricas exhiben un grado de contaminación de diversa índole.

La acuicultura es una actividad importante para el desarrollo productivo del sector agropecuario, participa en reducir la pobreza y en programas de seguridad alimentaria en Colombia (MADR, FAO e INCODER, 2011). Actividad que inicia en la década de 1930 con la introducción de Oncorhyncus mykiss, 20 años más tarde se introduce Oreochromis mossambicus.

En 1963 se inicia la construcción de la Estación Piscícola en la Granja Montelindo (vereda Santágueda, municipio de Palestina, Caldas, Colombia) dando origen al programa piscícola adscrito a la Facultad de Medicina Veterinaria y Zootecnia de la Universidad de Caldas. En 1967 la Universidad de Caldas introdujo 10 parejas de Tilapia rendalli (Tilapia rendalli) provenientes de Brasil con las que se principiaron los trabajos de policultivo con Tucunaré (Cichla ocellaris), Óscar (Astronotus ocellatus) y Mojarra amarilla (Petenia kraussi), y se comenzó el primer programa de piscicultura campesina en el país, en convenio con la Federación Nacional de Cafeteros y otras entidades gubernamentales. En la década de 1970 el Instituto de Recursos Naturales y Renovables (INDERENA) importó la 
Tilapia nilótica (Oreochromis niloticus), para programas de fomento. A mediados de 1980 se introdujo la Tilapia roja (Oreochromis spp.) al departamento del Valle del Cauca desarrollándose a partir de ahí la piscicultura comercial (Ruiz, 1990). En 1989, el régimen del Acuerdo Internacional del Café (ICA - International Coffee Agreement) se derrumba y el equilibrio de poder en la cadena global del café cambió claramente, afectando directamente los programas de diversificación (Ponte, 2002; Bitzer, Francken y Glasbergen, 2008) permaneciendo activo el programa de piscicultura hasta principios de los años noventa. A mediados de esta década la Universidad de Caldas realiza convenios con la Unión Europea explorando especies nativas como la Dorada (Brycon moorei), Bocachico (Prochilodus reticulatus) y Cachamas (Colossoma macropomum, Piaractus brachipomum).

Con base en el informe del MADR et al. (2011), que sostiene que "los desbalances entre la oferta y la demanda por el recurso hídrico no hacen prever problemas de desabastecimiento en el mediano plazo" (p. 19), la realidad de la Estación Piscícola de la Universidad de Caldas es distinta. La intensa deforestación de la región andina, adicionada a la intensificación de los cultivos perenes y transitorios, la excesiva fertilización y la contaminación del agua por lixiviados de urbanizaciones, incumpliendo las reglamentaciones sanitarias y ambientales, han ocasionado una disminución dramática de la cantidad y calidad del agua, problemática similar a otras estaciones piscícolas del país. La Conferencia de Rio+20 enfatiza sobre la necesidad de integrar los recursos hídricos como parte del desarrollo sostenible, lo que hace necesario tomar acciones para realizar modelos sustentables en la producción, preservar y optimizar el recurso natural base del desarrollo económico y social (Naciones Unidas, 2012b).

La optimización en el uso del recurso hídrico de la Estación Piscícola de la Universidad de Caldas obligó a buscar estrategias que permitieran la sostenibilidad de la misma. Es así como se opta por recircular (reutilizar) el agua como una alternativa viable para la estación, adaptando un mecanismo ancestral como es la rueda hidráulica o Noria. El nombre proviene posiblemente de la palabra árabe noria (na'oura) (Al-Dbiyat, 2009), saqiya (en árabe "el irrigador") (Mays, 2010a), na'ura (en árabe "ruidoso") y noriya (en árabe "ladrón, que roba el agua") en Israel, Transjordania y Siria: rueda persa; en Irán, India y Pakistán: rueda de accionamiento hidráulico (Ayalon, Milo y Nes, 2000).

Este artículo hace un recuento histórico del entorno ambiental de la microcuenca del caño El Berrión en la cual se asienta la Estación Piscícola, y como resultado a esta problemática la adaptación de un sistema de recirculación de agua usando una Noria modificada, proponiéndose como un modelo sostenible para la producción acuícola en países en desarrollo, coincidiendo con la tesis de que los conocimientos tradicionales son aplicables a una nueva era al incorporar una innovación dinámica, que permitan alcanzar la sostenibilidad local y medioambiental (Mays, 2010b).

La Universidad de Caldas, cuenta hace 52 años con el programa de producción piscícola en la Granja Montelindo, donde se realizan actividades académicas, de investigación y proyección, esta última con énfasis en piscicultura campesina. A partir de la década de 1980 se identificaron cambios en la microcuenca, afectando la cantidad y calidad de agua que abastece la Estación Piscícola. Es así como se observa la 
necesidad de implementar una estrategia de reutilización de agua, mediante el uso de una Noria modificada, que busca una solución amigable y sostenible con el ambiente.

\section{METODOLOGÍA}

\section{Área de estudio}

El trabajo se desarrolló en la Estación Piscícola de la Universidad de Caldas, localizada en la microcuenca del caño El Berrión, veredas Los Lobos y Santágueda, municipio de Palestina (Caldas, Colombia), coordenadas: Longitud W $75^{\circ} 45^{\prime}$ - Latitud N $5^{\circ} 04^{\prime}$ ) (Figura 1). En la Figura 2 se observa parte de la microcuenca El Berrión y la Estación Piscícola. La microcuenca presenta elevaciones entre los 1000 y $1400 \mathrm{msnm}$. Posee 541,3 ha, hace parte de la microcuenca de la quebrada Carminales, y esta a su vez de la cuenca del río Chinchiná (Cauca-Caribe) la cual superó el $10 \%$ de la presión sobre su recurso hídrico a partir del año 2008 (Grajales, 2004; CORPOCALDAS, 2007).

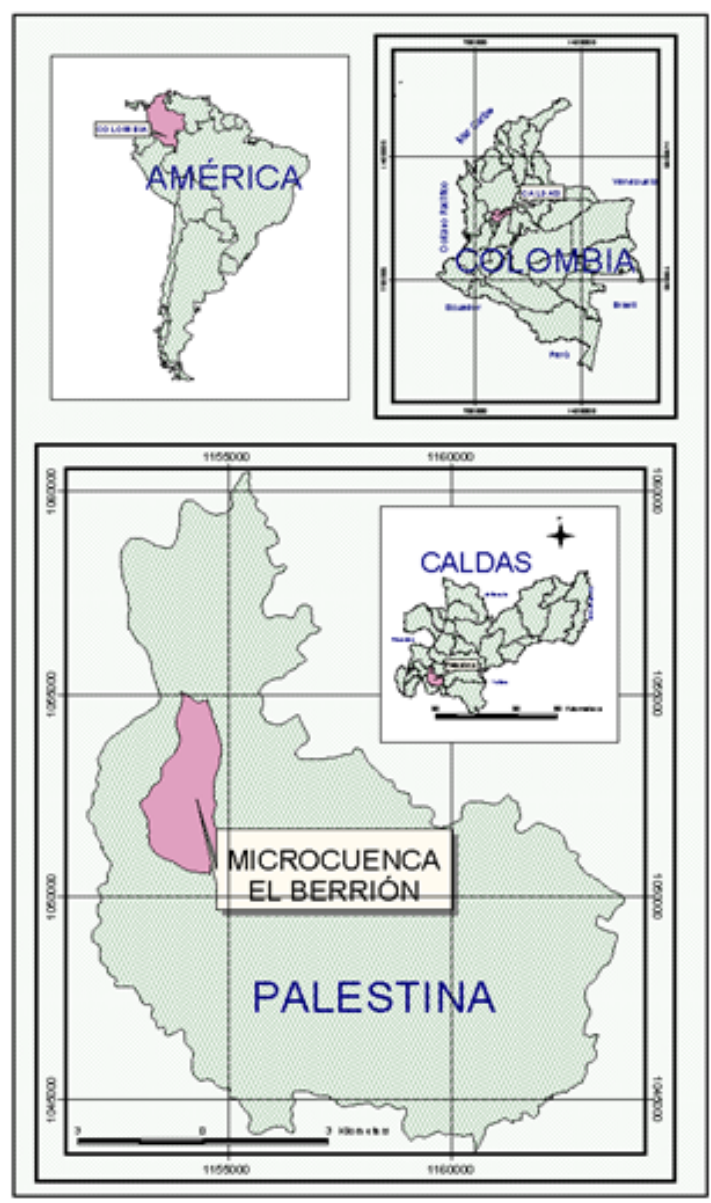

Figura 1. Localización geográfica de la microcuenca El Berrión. 


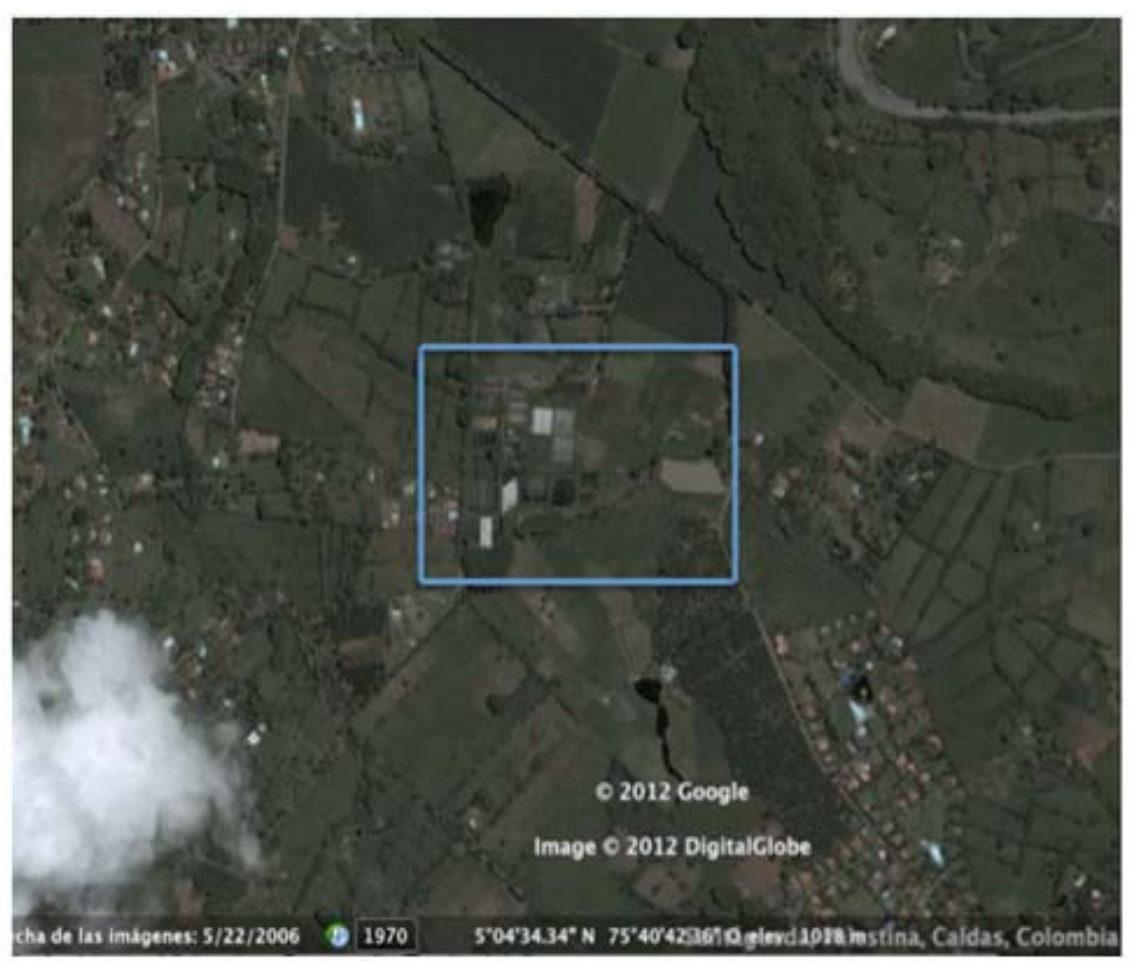

Fuente: imagen tomada Google Earth.

Figura 2. Microcuenca El Berrión, y Estación Piscícola de la Universidad de Caldas.

\section{Recuento ambiental del área de estudio}

La geología del sector se compone de depósitos inconsolidados de flujo de lodo; con fuertes precipitaciones produciendo el arrastre de material de la zona montañosa que actúa como área de recarga de acuíferos; presenta fenómenos erosivos por la deforestación y cultivos semestrales, los cuales se consideran inadecuados para preservar la estabilidad de laderas y disminuir el proceso erosivo. En el punto de inflexión entre alta pendiente y el valle se produce el depósito de sedimentos, afectando directamente la Estación Piscícola, y un cauce sinuoso y estrecho que abandona frecuentemente en épocas de lluvia. Esta zona ha sido ocupada por condominios y viviendas campestres, ocasionando desviación del cauce. La Estación es influenciada directamente por las corrientes de aire caliente de la cuenca del río Cauca y de las corrientes de aire frío procedentes del Nevado del Ruiz (Grajales, 2004). Presenta temperatura máxima de $33^{\circ} \mathrm{C}$, media de $22,6^{\circ} \mathrm{C}$ y mínima de $15^{\circ} \mathrm{C}$; altura sobre el nivel del mar: $1050 \mathrm{msnm}$; precipitación de $2300 \mathrm{~mm}$; comportamiento bimodal, humedad relativa 74\%; brillo solar: 2300 horas/año [Estación climatológica Santágueda (código 2615509), coordenadas 0505-N 7540, Fecha de Instalación 1964-01].

En 1963, cuando se comenzó la construcción de la Estación Piscícola, la parte alta de la microcuenca presentaba bosques secundarios y algunos pequeños cultivos, los cuales pasaron a cultivos de café arábigo asociado con árboles de sombrío (Kumar, 1978. Posteriormente, se desarrollaron variedades de café que no requieren sombrío, ocasionando la tala de árboles (Muschler y Bonneman, 1997), afectando la biodiversidad y causando impacto negativo sobre los nacimientos de agua. Se introducen al país patologías que afectan al café y se desarrollan planes de fomento 
para las zonas donde no se cultivaba el grano, desapareciendo en las zonas marginales (Buriticá, 2010). Se elimina entonces el café de la microcuenca del caño El Berrión, y los suelos cambiaron su cobertura hacia cultivos limpios y un marcado desarrollo turístico que afecta la cantidad y calidad del agua. Hoy la Estación Piscícola recibe en promedio $1,8 \mathrm{~L} / \mathrm{seg}$ de agua en verano y $15 \mathrm{~L} / \mathrm{seg}$ en invierno; las aguas de escorrentía se recogen en un reservorio de $6.000 \mathrm{~m}^{2}$, y posee un pozo profundo con una capacidad de generación $15 \mathrm{~L} / \mathrm{seg}$, cuya agua tiene en promedio un $\mathrm{pH}$ de 7,1 , temperatura de $24^{\circ} \mathrm{C}$ y oxígeno de $3,3 \mathrm{ppm}$. Se estima una pérdida del $20 \%$ de agua a lo largo del recorrido por la Estación.

La calidad de agua de la Estación Piscícola fue determinada mediante bioindicadores, analizando los macroinvertebrados acuáticos (Hahn-vonHessberg et al., 2009) encontrando 55 familias, siendo las más importantes Chironomidae $(32,5 \%)$ y Thiaridae $(26,7 \%)$ asociadas a bajos niveles de oxígeno, altas concentraciones de recuento de mesófilos, alta conductividad y altos niveles de microorganismos. Las demás familias tuvieron un $5 \%$ de representatividad, excepto la familia Palaemonidae con $6,7 \%$ de la población total. Los hongos registrados pertenecen a los grupos Oomycetos, Phycomycetos y Zygomicetos y morfoespecies como Cladosporium sp., Thielaviopsis sp., Rhizopus sp., Penicillium sp.

y Fusarium sp. Los estudios limnológicos identificaron organismos planctónicos

como Chroococcus sp., Botryococcus, Closterium, Coelastrum, Golenkini s p., Pediastrum sp., Scenedesmus sp., Spirogyra sp., Staurastrum, Ulothrix sp., Navicula sp., Synedra, Tabellaria, Asplachna sp., Brachionus sp., Filini a sp., Keratella sp., Lecane sp., Philodina sp., Trichocerca sp., Daphnia sp ., Copépodos, Ferrissia sp., Epistylis (Hahn-von-Hessberg et al., 2007). Los parámetros fisicoquímicos del agua de la Estación en diversos puntos de observación (canales de acceso, estanques, rebose de los estanques, canales recolectores, noria) dieron como resultado para la temperatura un promedio de $24^{\circ} \mathrm{C}+1,59^{\circ} \mathrm{C}$, oxígeno disuelto de $4,3+1,2 \mathrm{mg} / \mathrm{L}$ y $\mathrm{pH}$ de $7,2+0,45$.

\section{Diseño y elaboración del sistema de recirculación de agua}

La infraestructura existente en la Estación Piscícola se diseño acorde a las necesidades del campesino de zona andina donde predomina una topografía quebrada, poca cantidad y baja calidad de agua, en el que se han desarrollado modelos autóctonos y de recuperación, buscando la optimización de los recursos y minimizar costos. Los modelos de estanques han sido desarrollados en varias etapas, constan de 64 estanques (áreas unitarias entre 100 y $500 \mathrm{~m}^{2}$, con profundidad promedio de $1 \mathrm{~m}$, en tierra, cemento y tierra, cemento, polímero), un laboratorio ictiológico con $240 \mathrm{~m}^{2}$ de área cubierta. A partir de 1990 se recobra y moderniza la infraestructura existente, realizando nuevos diseños y materiales de recuperación para estanques de la zona andina.

El área utilizada para la recolección de agua de rebose o primera fase de recirculación es de $9.352 \mathrm{~m}^{2}$, la segunda fase comprenderá $6.200 \mathrm{~m}^{2}$ de un total de $21.832 \mathrm{~m}^{2}$ de espejo de agua que abarca la Estación. Para el desarrollo del proyecto de recirculación de agua no se realizaron modificaciones en el recorrido normal de abastecimiento de agua a los estanques ni de los canales de desagüe donde se obtiene el agua para su posterior recirculación (Figura 3 ). El recambio de agua en los estanques es únicamente de mantenimiento, devolviéndose normalmente $(80 \%$ 
promedio) al cauce natural de la quebrada. El sistema de recirculación desarrollado consta en su primera fase de la captación de agua de recambio de siete baterías de estanques, donde se construyeron cajas colectoras, adaptándolas con compuertas en la parte inferior y en la parte superior se adecúo una tubería de 3 pulgadas para conducir el agua hacia los compartimentos del cilindro, la cual hace parte de la Noria modificada, para así por medio de energía hidráulica impulsar esta a través de un sistema de poleas. La estructura en sí de la Noria consta de tres partes: una Noria compuesta por una rueda a la cual se le adaptaron canjilones e impulsada con una cadena de eslabones, un Cilindro con compartimentos donde se recolecta parte del agua y un sistema de Poleas (Figura 4).

La Noria modificada está localizada al final de un canal de desagüe, donde se recoge el agua de recambio de tres baterías de estanques que desaguan directamente, y el agua de cuatro baterías de estanques que caen previamente en el Cilindro con compartimentos para generar movimiento con las poleas (Figura 3 ). La Noria es una estructura circular de $7 \mathrm{~m}$ de diámetro, construido de forma modular con ángulos de hierro ajustados entre sí con tornillería, adaptada con 32 canjilones construidos en fibra de vidrio, cada uno con una capacidad de $5 \mathrm{~L}$, los cuales recolectan el agua cuando están en la parte inferior de la Noria, tomando únicamente la parte superficial del agua de la canal, evitando captar sedimentos; es llevado posteriormente el agua a la parte superior de la Noria donde los canjilones se desocupan y depositan el agua en la bandeja receptora, comenzando así la recirculación con un promedio de $1,5 \mathrm{~L} / \mathrm{seg}$ de agua, esta es conducida inicialmente por un tubo galvanizado, que a su vez sirve de soporte de la Noria (Figura 4), a continuación es transportada por gravedad hasta la parte superior del canal principal de abastecimiento de agua de la Estación a la altura del Laboratorio Ictiológico, completándose así el ciclo.

El diseño de la Noria modificada fue realizado teniendo en cuenta las mediciones correspondientes a la cantidad de agua a ser recirculada procedente de las salidas de las baterías de estanques, tanto en la época de verano como en invierno; además, determinado por los niveles del terreno, adaptándose a una infraestructura existente en la Estación y diseñado en forma modular, para aplicarlo a diferentes terrenos y necesidades donde se requiere recircular agua por medio de la gravedad sin la utilización de energía eléctrica. 


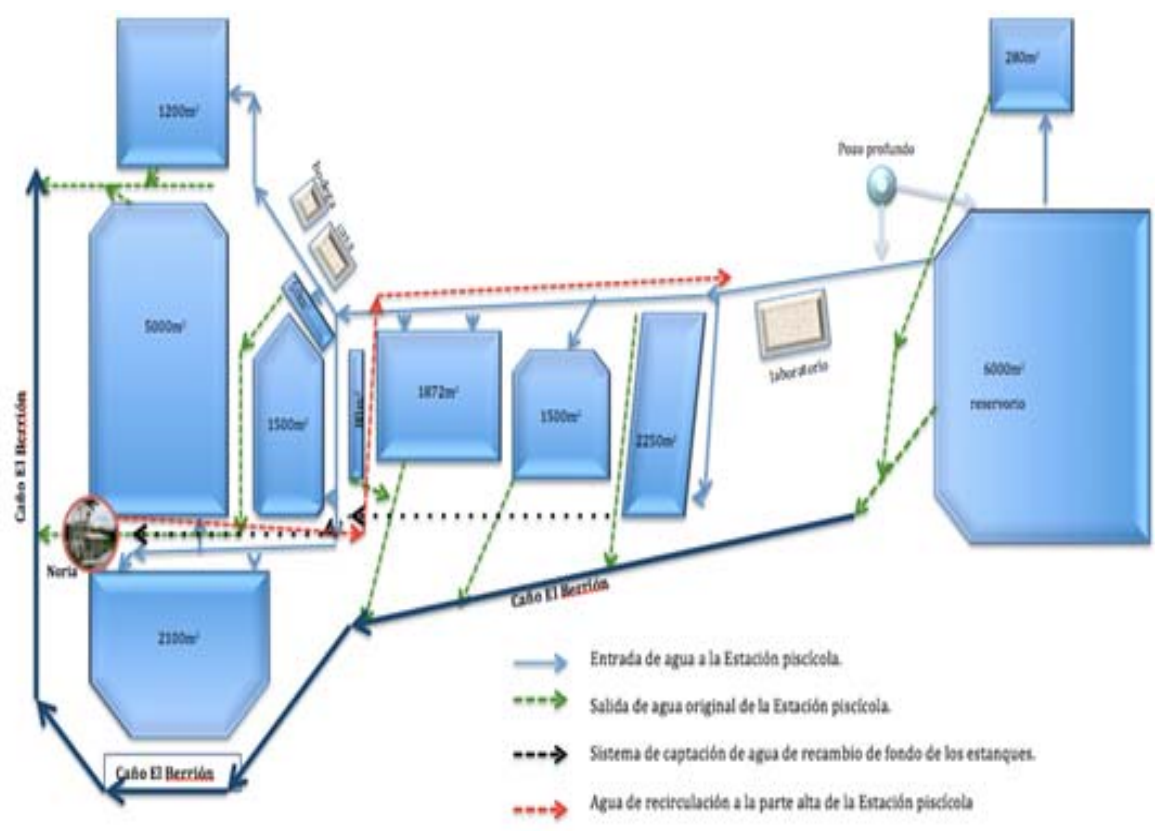

Figura 3. Plano general del sistema de recirculación de agua con la Noria modificada de la Estación Piscícola de la Universidad de Caldas.

Haga clic sobre la imagen para ampliarla 


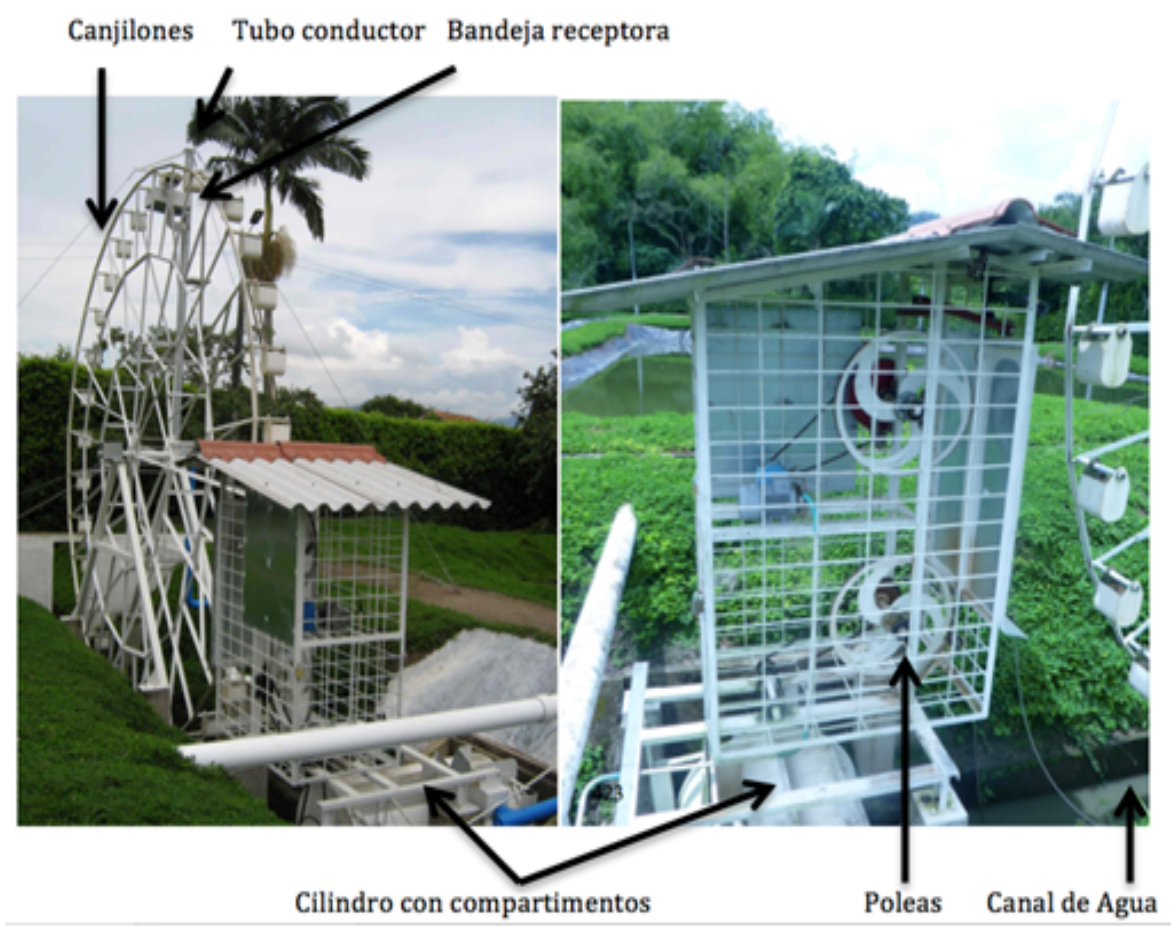

Figura 4. En la fotografía de la izquierda la Noria modificada con los canjilones,

bandeja receptora del agua y el tubo de sostén de la Noria y a su vez conductor de

agua a recircular, en la parte anterior la caseta donde se localiza el sistema de poleas.

En la fotografía de la derecha la caseta con el sistema de poleas, la cadena de

eslabones para el impulso de la Noria, y en la parte inferior el Cilindro con

compartimentos.

El Cilindro posee 8 compartimientos, cada uno con una capacidad de $60 \mathrm{~L}$, recibe el agua de recambio de $5.702 \mathrm{~m}^{2}$ de espejo de agua proveniente de cuatro baterías de estanques. Una vez que un compartimiento se ha llenado de agua, su peso produce un giro que induce un movimiento a través de las poleas conectado con una cadena de eslabones e impulsa la Noria, elevando los canjilones a la parte superior de la misma; la rueda se moverá dependiendo de la rapidez y la cantidad de agua que recibe el Cilindro con compartimentos. El sistema de poleas de la Noria está conformado por dos platos de hierro forjado de $70 \mathrm{~cm}$ de diámetro, conectados con la Noria y el Cilindro con compartimentos por medio de cadenas de eslabón permitiendo así su interacción.

En la Tabla 1 se discriminan los costos de construcción del sistema de recirculación de agua, como se ha descrito anteriormente. Los costos se han dividido en costos de construcción y mano de obra. Para la instalación se debe considerar una persona capacitada de apoyo a los trabajadores no cualificados. Los costos de ingeniería para la asesoría hidráulica no están incluidos. Los costos de materiales se indican en dólares. Se considera una vida útil calculada de 30 años, con un mantenimiento anual de $\$ 150$ dólares, puede trabajar 24 horas durante los 365 días del año y no posee consumo de energía eléctrica. 
Tabla 1. Costos de construcción del sistema de recirculación de agua

\begin{tabular}{|c|c|c|}
\hline & \multicolumn{2}{|c|}{$\begin{array}{r}\text { Costos de construcción del sistema de } \\
\text { recirculación de agua }\end{array}$} \\
\hline & Costo dólar-\$ & Porcentaje \\
\hline $\begin{array}{c}\text { Noria modificada, cilindro con } \\
\text { compartimentos y Poleas }\end{array}$ & 10.870 & $62,5 \%$ \\
\hline Redes hidráulicas & 3.043 & $17,5 \%$ \\
\hline Mano de obra & 3.479 & $20 \%$ \\
\hline Total & 17.392 & $100 \%$ \\
\hline
\end{tabular}

\section{RESULTADOS Y DISCUSIÓN}

Para el siglo XXI, el mundo se enfrenta a un aumento en la frecuencia de eventos extremos asociados a la variabilidad climática (Birkmann, Tetzlaff y Zentel, 2009); cerca del $50 \%$ de la tierra ha sido transformada, trayendo consecuencias funestas para la biodiversidad y el medio ambiente, y como consecuencia efectos sociales y económicos negativos (FAO, 2012b). Se plantean desafíos para el sector agropecuario, lo cual requiere incorporar estrategias de adaptación y tecnologías, que faciliten un desarrollo sostenible y anticiparse a los eventos hidroclimatológicos (Ocampo, 2012). A pesar de los esfuerzos generales para crear un futuro sostenible, los indicadores mundiales señalan que la humanidad se aleja de la sostenibilidad.

En Colombia se estimó para la década del 2000 una pérdida promedio anual de la cobertura boscosa de 273.334 ha/año para las regiones de la Amazonía y los Andes (Cabrera et al., 2011), afectando entre 2010-2011 el $60 \%$ de bosques primarios de alta complejidad (Naciones Unidas, 2012b). El agua es un recurso indispensable para el desarrollo económico y el bienestar social. En promedio se requieren $3.000 \mathrm{~L}$ de agua por persona para generar los productos necesarios para su alimentación diaria (Silva et al., 2008). Las actividades agrícolas requieren en promedio un $10 \%$ del agua usada (FAO y FIDA, 2006). Ante el crecimiento de la población, tipos de asentamientos, actividades productivas y sistemas tecnológicos, se presentan efectos como la desregulación de la disponibilidad espacial y temporal en la oferta hídrica, deterioro de las condiciones biológicas y fisicoquímicas del agua, conflictos intersectoriales e interterritoriales, lo que ha forzado a buscar nuevas fuentes de abastecimiento de agua considerándose las aguas residuales o usadas una alternativa de producción (Silva, Torres y Madera, 2008).

La cuenca del río Chinchiná, a la cual pertenece la microcuenca El Berrión, es afectada por el fenómeno de variabilidad climática natural ENSO-Oscilación del Sur; La Niña ocasiona un incremento en los caudales del $28 \%$ en promedio; El Niño produce una disminución en promedio del $24 \%$. Los cambios de caudal se deben a variaciones en las precipitaciones que producen los episodios fríos -aumentos del $27 \%$ al $140 \%$ - y los eventos cálidos -disminución del 18\% al 55\%- (IDEAM, 2010; Ocampo, 2012). Se estiman reducciones en la precipitación del $10 \%$ y aumento en la temperatura de $1,4^{\circ} \mathrm{C}$, presentándose una reducción en la 
oferta hídrica entre el $5 \%$ y el $28 \%$; el índice del uso del agua alcanzaría valores altos debido al crecimiento poblacional de la zona, las actividades económicas, la baja capacidad de regulación hídrica, manifestando un aumento considerable del Índice de Vulnerabilidad (Ocampo, 2012); coincidiendo con Pabón (2006), quien sugiere que se espera una disminución de los volúmenes de precipitación anuales hasta en un $30 \%$, con posibles aumentos de temperatura en promedio de $4^{\circ} \mathrm{C}$, en la región Caribe y Andina, afectando las vertientes de la zona Andina (PNUMA, 2006) y por ende la Estación.

El panorama planteado obligó a buscar alternativas, donde los problemas de cantidad y calidad de agua de la Estación puedan resolverse utilizando métodos tradicionales empleados durante cientos de años. El significado de la sostenibilidad en los tiempos modernos debe ser reevaluado a la luz de antiguas obras públicas y prácticas de gestión, sin embargo las tendencias actuales del uso del agua con tecnologías tradicionales cada día se están olvidando, poco se realiza para mantener y mejorarlas, para enfrentar el crecimiento de la población, la desertificación y los aspectos económicos. A nivel internacional, la inversión en investigación, evaluación, mantenimiento y despliegue de tecnologías tradicionales, ha sido básicamente inexistente (Mays, 2010b).

Es así como se desarrolló una Noria modificada como alternativa sostenible, para la recirculación de agua en producción piscícola; entendiéndose como sostenible, la capacidad de utilizar agua en cantidades suficientes para satisfacer las necesidades del ser humano, seguridad alimentaria y garantizar la restauración y mantenimiento de los ecosistemas para preservar la vida para las generaciones futuras (Mays, Koutsoyiannis y Angelakis, 2007), esta sostenibilidad del agua debe ser considerada en políticas de nivel local, regional, nacional y a nivel internacional (FAO, 2012a, b).

\section{CONCLUSIONES}

Se presentan las bondades de un proyecto exitoso de recirculación de agua, el cual responde a las necesidades de una Estación Piscícola integrando el recurso hídrico al desarrollo sostenible social, ambiental y económicamente rentable, como lo sugiere Naciones Unidas (2012a, b).

La microcuenca que abastece la Estación Piscícola sufre el deterioro propio de la zona Andina como es la intervención, deforestación y contaminación. El objetivo de este trabajo fue realizar un recuento de las condiciones ambientales y buscar la implementación de una tecnología milenaria como es la Noria, modificando su diseño y adaptándola a las necesidades locales para promover el uso sostenible y racional del agua.

Para completar el sistema de recirculación de agua, se recomienda acoplarlo a un sistema de tapetes biológicos que mejoren la calidad del agua y, de esta manera, lograr independencia de los factores externos de presión antrópica, como los asentamientos urbanos, cambio del uso de los suelos, pudiendo ser una solución para estaciones piscícolas o producciones agropecuarias. 
La academia juega un papel clave al iniciar el enfoque y el fomento en pro de nuevas alternativas a partir de tecnologías milenarias, buscando así alternativas productivas de bajo impacto ambiental y económicamente aplicables al campo.

\section{AGRADECIMIENTOS}

A la Secretaría de Agricultura de la Gobernación de Caldas, al Comité Departamental de Cafeteros de Caldas y a la Universidad de Caldas. Especial mérito al Señor Enrique Vélez Jaramillo, que con su ingenio y perseverancia fue posible realizar la Noria.

\section{REFERENCIAS}

- Al-Dbiyat M. (2009). Les Norias de Hama sur l'Oronte: un Système Traditionnel Original de L'Utilisation de l'eau fluviale gestion durable et équitable de l'eau douce en Méditerranée. Mémoire et Traditions avenir et solutions, Vèmes Rencontres Internationales Monaco et la Méditerranée Actes. Association Monégasque pour la Connaissance des Arts, 191-210.

- Ayalon, E., Milo, U. y Nes, Z. (2000). Typology and Chronology of WaterWheel ("sāqiya"). Israel Exploration Journal, 50(3/4), 216-226.

- Birkmann, J., Tetzlaff, G. y Zentel, K. O. (2009). Addressing the challenge: recommendations and quality criteria for linking disaster risk reduction and adaptation to climate change. En Birkmann, J., Tetzlaff, G., Zentel y KarlOtto (Eds.). DKKV Publication Series 38. Bonn.

- Bitzer, V., Francken, M. y Glasbergen, P. (2008). Intersectoral partnerships for a sustainable coffee chain: Really addressing sustainability or just picking (coffee) cherries? Global Environmental Change, 18, 271-284.

- Buriticá C., P. (2010). The Coffee Rust in Colombia: Achievements of National and International Impact in the Twentieth Century. Revista Facultad Nacional Agronomía, Medellín, 63(1), 5285-5292.

- Cabrera E., Vargas, D. M., Galindo, G., García, M. C. y Ordóñez, M. F. (2011). Memoria Técnica: Cuantificación de la tasa de deforestación para Colombia, Periodo 1990-2000, 2000-2005. Instituto de Hidrología, Meteorología y Estudios Ambientales -IDEAM-, Bogotá, D.C., Colombia.

- Corporación Autónoma Regional de Caldas -CORPOCALDAS-. (2006). El Papel de Corpocaldas como Autoridad Ambiental dentro de la Gestión Local del Riesgo. Taller Internacional sobre Gestión del Riesgo a Nivel Local, El Caso de Manizales, Colombia la Administración Pública y el rol de la Universidad. Manizales: Una Ciudad Laboratorio. Manizales, Septiembre 2006.

- Corporación Autónoma Regional de Caldas -CORPOCALDAS-. (2007). Plan de gestión ambiental regional 2007-2019 (Documento interno de trabajo, Corpocaldas - Agosto 2007).

- Grajales, A. (2004). Evaluación de dos tipos de resolución de los SIG, utilizando la variabilidad espacial de la Erodabilidad del suelo en la Microcuenca "El Berrión", Municipio de Palestina, Caldas, Colombia. Tesis de Magíster, Facultad de Ciencias Agropecuarias, Universidad de Caldas. Manizales, Colombia. 
- Hahn-von-Hessberg, C. M., Toro, D. R., Grajales Q., A. y Henao C., A. F. (2007). Producción limnológica en estanques para el levante de larvas y postlarvas de especies nativas y foráneas, Caldas, Colombia. Revista Electrónica de Ingeniería en Producción Acuícola, 2(2), 137-168.

- Hahn-von-Hessberg, C. M., Toro, D. R., Grajales, Q., A., Duque, G. M., y Serna, U. L. (2009). Determinación de la Calidad del Agua Mediante Indicadores Biológicos y Fisicoquímicos, en la Estación Piscícola, Universidad de Caldas, Municipio de Palestina, Colombia. Bol. Cient. Mus. Hist. Nat. U. de Caldas, 13(2), 89-105.

- Instituto de Hidrología, Meteorología y Estudios Ambientales -IDEAM-. (2010). Estudio Nacional de Agua 2010. Bogotá: Ministerio de Medio Ambiente, Vivienda y Desarrollo Territorial.

- Kumar, D. (1978). Investigation into some physiological aspects of high density plantings of coffee (Coffea arabica L.). Coffee Kenya, 43(510), 263-272.

- Mays, L. W. (2010a). Water Technology in Ancient Egypt. Chapter 3. En WT in ancient Egypt (pp. 53-65).

- Mays, L. W. (2010b). Lessons from the Ancients on Water Resources Sustainability. Chapter 11. En Lessons from the Ancients on Water resources sustainability (pp. 217-239).

- Mays, L. W., Koutsoyiannis, D. y Angelakis, A. N. (2007). A brief history of urban water distribution. Water Science and Technology: Water Supply, 7(1), 1-12.

- Ministerio de Agricultura y Desarrollo Rural -MADR-, FAO e INCODER. (2011). Plan Nacional de Desarrollo de la Acuicultura Sostenible en Colombia. Diagnóstico del Estado de la Acuicultura en Colombia, Diciembre de 2011.2 Recuperado de http://www.ceniacua.org/archivos/Diagnostico para revision Dic 520 11 v1.pdf

- Ministerio de Ambiente, Vivienda y Desarrollo Territorial -MAVDT-. (2010). Segunda Comunicación Nacional ante la Convención Marco de las Naciones Unidas sobre Cambio Climático, República de Colombia. Junio de 2010. Bogotá, D.C.: IDEAM. ISBN: 978-958-8067-31-5. Recuperado de https://documentacion.ideam.gov.co/openbiblio/Bvirtual/021658/2Comu nicacion/IDEAMTOMOIIPreliminares.pdf

- Ministerio de Ambiente, Vivienda y Desarrollo Territorial -MAVDT- y Viceministerio de Ambiente. (2010). Política Nacional para la Gestión Integral del Recurso Hídrico. Bogotá, D.C.: Ministerio de Ambiente, Vivienda y Desarrollo Territorial. ISBN: 978-958-8491-35-6. Recuperado de https://www.minambiente.gov.co/images/GestionIntegraldelRecursoHidr ico/pdf/Presentaci\%C3\%B3n Pol\%C3\%ADtica Nacional Gesti\%C3\%B3 n /libro pol nal rec hidrico.pdf

- Muschler, R. G. y Bonnemann A. (1997). Potentials and limitations of agroforestry for changing land-use in the tropics: experiences from Central America. Forest Ecology and Management, 19, 61-73.

- Naciones Unidas. (2012a). Colombia. Monitoreo de Cultivos de Coca 2011. UNDOC. Oficina de las Naciones Unidas contra la Droga y el Delito. ISSN - 2011-0596.Recuperado de https://www.unodc.org/documents/cropmonitoring/Colombia/Colombia Monitoreo de Cultivos de Coca $2012 \mathrm{w}$ eb.pdf

- Naciones Unidas. (2012b). Conferencia de las Naciones Unidas sobre el desarrollo sostenible. Río de Janeiro (Brasil) 20 a 22 de junio de 2012. A/CONF.216/L.1.

Recuperado de http://www.uncsd2012.org/content/documents/778futurewewant spanis $\underline{\text { h.pdf }}$ 
- Ocampo L., O. L. (2012). Análisis de vulnerabilidad de la cuenca del río Chinchiná para condiciones estacionarias y de cambio climático. Tesis de Magíster, Facultad de Ingeniería, Universidad Nacional de Colombia. Colombia.

- Organización de las Naciones Unidas para la Alimentación y la Agricultura -FAO- y Fondo Internacional de Desarrollo Agrícola -FIDA-. (2006). El agua para la alimentación, la agricultura y los medios de vida rurales. En El agua, una responsabilidad compartida. $2^{\circ}$ Informe de las Naciones Unidas sobre el desarrollo de los recursos hídricos en el mundo. Recuperado de http://www.unesco.org/water/wwap/index_es.shtml

- Organización de las Naciones Unidas para la Alimentación y la Agricultura. FAO. (2012a). The State of World Fisheries and Aquaculture 2012. Recuperado de http://www.fao.org/docrep/016/i2727e/i2727e.pdf

- Organización de las Naciones Unidas para la Alimentación y la Agricultura. FAO. (2012b). Voluntary Guidelines on the Responsible Governance of Tenure of Land, Fisheries and Forests in The Context of National Food Security. Rome. Recuperado de http://www.fao.org/docrep/016/i2801e/i2801e.pdf

- Pabón, J. D. (2006). Escenarios de cambio climático para Colombia. En Memorias del IV Encuentro de la Red de Universidades del Pacífico Sur (RUPSUR), 8-10 de noviembre de 2006. Recuperado de http://www.idea.unal.edu.co/eventos/CisdalV/ponencias/E2 Cambio cli ma/E2 daniel pabon.pdf

- Ponte, S. (2002). The 'latte revolution'? Regulation, markets and consumption in the global coffee chain. World Development, 30, 10991122.

- Programa de las Naciones Unidas para el Medio Ambiente -PNUMA-. (2006). El Cambio Climático en América Latina y el Caribe. México: Secretaría de Medio Ambiente y Recursos Naturales -SEMARNAT-. ISBN 968-817-677-X.

Recuperado de http://www.oei.es/decada/ElcambioClimatico r.pdf

- Quintero-Ángel, M., Carvajal-Escobar, Y. y Aldunce, P. (2012). Adaptación a la variabilidad en el cambio climático: intersecciones con la gestión del riesgo. Revista Luna Azul, 34, 257-271. Recuperado de http://lunazul.ucaldas.edu.co/index.php?option=content\&task=view\&id= $\underline{706}$

- Ruiz R., L. E. (1990). Colombia y la acuicultura. Instituto Nacional de los Recursos Naturales Renovables y del Ambiente -INDERENA-. Bogotá. Recuperado de http://www.fao.org/docrep/005/ad020s/AD020s04.htm

- Silva, J., Torres, P. y Madera, C. (2008). Reuso de aguas residuales domésticas en agricultura. Agronomía Colombiana, 26(2), 347-359.

1. Esp. (c)Msc. Docente, Departamento de Producción Agropecuaria, Universidad de Caldas. Manizales, Colombia. christine.hahn@ucaldas.edu.co

2. Ph. Docente Investigador, Pesquerías y Acuacultura, Universidad British Columbia - Canadá. qfherbert@gmail.com

3. Ph. Docente, Departamento de Producción Agropecuaria, Universidad de Caldas. Manizales, Colombia. alberto.grajales@ucaldas.edu.co 
Para citar este artículo: Hahn-von-Hessberg, C. M., Quintero, H. E. y Grajales-Quintero, A. (2016). Desarrollo e implementación de una Noria modificada como propuesta sostenible de recirculación de agua para una estación piscícola. Revista Luna Azul, 42, 185-199. Recuperado de http://200.21.104.25/lunazul/index.php?option=com_content\&view=article\& id $=134$ 